\title{
APPLICATIONS OF ANDREWS' BASIC LAURICELLA TRANSFORMATION
}

\author{
D. M. BRESSOUD
}

\begin{abstract}
We view Andrews' transformation for the fourth basic Lauricella function as a multiple summation analog of Heine's fundamental transformation for ${ }_{2} \phi_{1}$. This leads to multiple summation analogs of several classical $q$-series identities, and a new proof of a recent partition theorem.
\end{abstract}

Introduction. We consider two special cases of Andrews' transformation for the fourth basic Lauricella function, and demonstrate how these can be used to generalize known $q$-series identities. We shall also use one of these results to prove a partition identity.

The fourth basic Lauricella function is defined as:

$$
\begin{aligned}
\Phi_{D}\left[\alpha ; \beta_{1}, \ldots, \beta_{n} ; \gamma ; q ; x_{1}, \ldots, x_{n}\right] \\
\quad=\sum_{m_{1}, \ldots, m_{n}>0} \frac{(\alpha)_{M}\left(\beta_{1}\right)_{m_{1}} \cdots\left(\beta_{n}\right)_{m_{n}} x_{1}^{m_{1}} \cdots x_{n}^{m_{n}}}{(q)_{m_{1}} \cdots(q)_{m_{n}}(\gamma)_{M}},
\end{aligned}
$$

where $M=m_{1}+m_{2}+\cdots+m_{n}$ and $(A)_{m}=(A ; q)_{m}=(1-A)(1-$ $A q)\left(1-A q^{2}\right) \cdots\left(1-A q^{m-1}\right)$. The generalized basic hypergeometric function is

$$
{ }_{r} \phi_{s}\left[\begin{array}{c}
a_{1}, \ldots, a_{r} ; q, x \\
b_{1}, \ldots, b_{s}
\end{array}\right]=\sum_{m>0} \frac{\left(a_{1}\right)_{m}\left(a_{2}\right)_{m} \cdots\left(a_{r}\right)_{m} x^{m}}{(q)_{m}\left(b_{1}\right)_{m} \cdots\left(b_{s}\right)_{m}} .
$$

Andrews [4] has shown that:

$$
\begin{aligned}
& \Phi_{D}\left[\alpha ; \beta_{1}, \ldots, \beta_{n} ; \gamma ; q ; x_{1}, \ldots, x_{n}\right] \\
& \quad=\frac{(\alpha)_{\infty}}{(\gamma)_{\infty}} \prod_{i=1}^{n} \frac{\left(\beta_{i} x_{i}\right)_{\infty}}{\left(x_{i}\right)_{\infty}}{ }_{n+1} \phi_{n}\left[\begin{array}{c}
\gamma / \alpha, x_{1}, x_{2}, \ldots, x_{n} ; q, \alpha \\
\beta_{1} x_{1}, \ldots, \beta_{n} x_{n}
\end{array}\right] .
\end{aligned}
$$

This is a generalization of Heine's fundamental transformation for ${ }_{2} \phi_{1}[9, p$. 106]:

$$
{ }_{2} \phi_{1}\left[\begin{array}{c}
\alpha, \beta ; q, x \\
\gamma
\end{array}\right]=\frac{(\alpha)_{\infty}(\beta x)_{\infty}}{(\gamma)_{\infty}(x)_{\infty}}{ }_{2} \phi_{1}\left[\begin{array}{c}
\gamma / \alpha, x ; q, \alpha \\
\beta x
\end{array}\right] .
$$

Andrews has found applications of (1) [2, pp. 208-213], most of which involve the fourth basic Lauricella function with $\beta_{2}=x_{1} / x_{2}, \beta_{3}=x_{2} / x_{3}, \ldots, \beta_{n}=$ $x_{n-1} / x_{n}$.

Received by the editors June 6, 1977.

AMS (MOS) subject classifications (1970). Primary 33A30; Secondary 10A45, 05 A17.

Key words and phrases. Basic hypergeometric function, basic Lauricella function, $q$-series, Kummer's Theorem, partition. 
Recently, Andrews and Askey [5] announced new proofs of known $q$-series identities, showing these identities to be consequences of equation (2). We shall imitate their proofs of two of Rogers' identities, substituting equation (1) for equation (2) in order to obtain generalizations.

Rogers' two identities are

$$
\begin{aligned}
\sum_{n>0} \frac{q^{n^{2}} a^{n}}{(q)_{n}} & =\left(-a q^{2} ; q^{2}\right)_{\infty} \sum_{n>0} \frac{q^{n^{2}} a^{n}}{\left(q^{2} ; q^{2}\right)_{n}\left(-a q^{2} ; q^{2}\right)_{n}}, \\
\sum_{n>0} \frac{q^{4 n^{2}} a^{2 n}}{\left(q^{4} ; q^{4}\right)_{n}} & =\left(a q ; q^{2}\right)_{\infty} \sum_{n>0} \frac{q^{n^{2}} a^{n}}{\left(q^{2} ; q^{2}\right)_{n}\left(a q^{2} ; q^{2}\right)_{n}} .
\end{aligned}
$$

These appear in slightly different form in [10, p. 330]. Watson [11, pp. 46-47] has also proved (3) and (4), and Andrews has generalized these identities in a different direction [3, (15), (16)].

Our generalizations of (3) and (4) are, respectively:

$$
\begin{aligned}
& \sum_{n>0} \frac{q^{n+k n(n-1) / 2} a^{n}}{(q)_{n}} \\
& =\left(-a q^{k} ; q^{k}\right)_{\infty} \sum_{n_{1}, \ldots, n_{k-1}>0} \frac{a^{N} q^{k N(N-1) / 2+n_{1}+2 n_{2}+\cdots+(k-1) n_{k-1}}}{\left(q^{k} ; q^{k}\right)_{n_{1}} \cdots\left(q^{k} ; q^{k}\right)_{n_{k-1}}\left(-a q^{k} ; q^{k}\right)_{N}} \\
& \left(N=n_{1}+n_{2}+\cdots+n_{k-1}\right) \text {, } \\
& \sum_{m>0} \frac{q^{k^{2} m^{2}} a^{k m}}{\left(q^{2 k} ; q^{2 k}\right)_{m}} \\
& =\left(-a q ; q^{2}\right)_{\infty} \sum_{m_{1}, \ldots, m_{k-1}>0} \frac{q^{M^{2}} a^{M \zeta^{m_{1}}+2 m_{2}+\cdots+(k-1) m_{k-1}}}{\left(q^{2} ; q^{2}\right)_{m_{1}} \cdots\left(q^{2} ; q^{2}\right)_{m_{k-1}}\left(-a q ; q^{2}\right)_{M}} \\
& \left(M=m_{1}+m_{2}+\cdots+m_{k-1} ; \zeta=e^{2 \pi i / k}\right) \text {. }
\end{aligned}
$$

In proving (5) and (6), we shall also obtain generalizations of the $q$-analog of Kummer's Theorem and a theorem of Gauss.

Proof OF (5).

Proposition 1.

$$
\begin{aligned}
\Phi_{D}\left[b^{k} ;\right. & \overbrace{}^{k} / c, \ldots, b^{k} / c ; c ; q^{k} ; c q^{2} / b^{k}, \ldots, c q^{k-1} / b^{k}] \\
= & \frac{1}{k} \frac{(q)_{\infty}}{\left(c q / b^{k}\right)_{\infty}} \frac{\left(c q^{k} / b^{k} ; q^{k}\right)_{\infty}}{\left(c ; q^{k}\right)_{\infty}\left(q^{k} ; q^{k}\right)_{\infty}} \\
& \times \sum_{j=0}^{k-1}\left(c \zeta^{j} / b^{k-1}\right)_{\infty}(b)_{\infty}(\zeta b)_{\infty} \cdots(\widehat{\zeta} \hat{b})_{\infty} \cdots\left(\zeta^{k-1} b\right)_{\infty},
\end{aligned}
$$

where $\zeta=e^{2 \pi i / k}$. 
Proof. In (1), let $n=k-1$, replace $q$ by $q^{k}$ and set $\alpha=b^{k}, \beta_{1}=\beta_{2}$ $=\cdots=\beta_{k-1}=b^{k} / c, \gamma=c, x_{1}=c q / b^{k}, x_{2}=c q^{2} / b^{k}, \ldots, x_{k-1}=$ $c q^{k-1} / b^{k} ;$ then

$$
\begin{aligned}
& \Phi_{D}\left[b^{k} ; b^{k} / c, \ldots, b^{k} / c ; c ; q^{k} ; c q / b^{k}, c q^{2} / b^{k}, \ldots, c q^{k-1} / b^{k}\right] \\
& =\frac{\left(b^{k} ; q^{k}\right)_{\infty}}{\left(c ; q^{k}\right)_{\infty}} \prod_{i=1}^{k-1} \frac{\left(q^{i} ; q^{k}\right)_{\infty}}{\left(c q^{i} / b^{k} ; q^{k}\right)_{\infty}} \\
& \times_{k} \phi_{k-1}\left[\begin{array}{c}
c / b^{k}, c q / b^{k}, \ldots, c q^{k-1} / b^{k} ; q^{k} ; b^{k} \\
q, q^{2}, \ldots, q^{k-1}
\end{array}\right] \\
& =\frac{\left(b^{k} ; q^{k}\right)_{\infty}}{\left(c ; q^{k}\right)_{\infty}} \frac{(q)_{\infty}}{\left(c q / b^{k}\right)_{\infty}} \frac{\left(c q^{k} / b^{k} ; q^{k}\right)_{\infty}}{\left(q^{k} ; q^{k}\right)_{\infty}} \sum_{n>0} \frac{\left(c / b^{k}\right)_{k n}}{(q)_{k n}} b^{k n} \\
& =\frac{\left(b^{k} ; q^{k}\right)_{\infty}}{\left(c ; q^{k}\right)_{\infty}} \frac{(q)_{\infty}}{\left(c q / b^{k}\right)_{\infty}} \frac{\left(c q^{k} / b^{k} ; q^{k}\right)_{\infty}}{\left(q^{k} ; q^{k}\right)_{\infty}} \\
& \times \frac{1}{k} \sum_{n>0} \frac{\left(c / b^{k}\right)_{n}}{(q)_{n}} b^{n} \sum_{j=0}^{k-1} \zeta^{j n} \\
& =\frac{1}{k} \frac{\left(b^{k} ; q^{k}\right)_{\infty}}{\left(c ; q^{k}\right)_{\infty}} \frac{(q)_{\infty}}{\left(c q / b^{k}\right)_{\infty}} \frac{\left(c q^{k} / b^{k} ; q^{k}\right)_{\infty}}{\left(q^{k} ; q^{k}\right)_{\infty}} \sum_{j=0}^{k-1} \frac{\left(c \zeta^{j} / b^{k-1}\right)_{\infty}}{\left(\zeta^{j} b\right)_{\infty}}
\end{aligned}
$$

(by Bailey, [6, p. 66, (4)])

$$
\begin{aligned}
= & \frac{1}{k} \frac{(q)_{\infty}}{\left(c q / b^{k}\right)_{\infty}} \frac{\left(c q^{k} / b^{k} ; q^{k}\right)_{\infty}}{\left(c ; q^{k}\right)_{\infty}\left(q^{k} ; q^{k}\right)_{\infty}} \\
& \times \sum_{j=0}^{k-1}\left(c \zeta^{j} / b^{k-1}\right)_{\infty}(b)_{\infty}(\zeta b)_{\infty} \cdots\left(\widehat{\zeta^{j} b}\right)_{\infty} \cdots\left(\zeta^{k-1} b\right)_{\infty} .
\end{aligned}
$$

This establishes Proposition 1.

Corollary 1.1 .

$$
\begin{aligned}
\sum_{m_{1}=0}^{s} \sum_{m_{2}=0}^{s-m_{1}} & \cdots \sum_{m_{k-1}=0}^{s-\left(m_{1}+m_{2}+\cdots+m_{k-2}\right)} \\
& \times \frac{q^{m_{1}+2 m_{2}+\cdots+(k-1) m_{k-1}}}{\left(q^{k} ; q^{k}\right)_{s-\left(m_{1}+\cdots+m_{k-1}\right)}\left(q^{k} ; q^{k}\right)_{m_{1}} \cdots\left(q^{k} ; q^{k}\right)_{m_{k-1}}} \\
= & \frac{1}{(q)_{s}} .
\end{aligned}
$$

Proof. In Proposition 1 , set $b=q^{-s}$ and let $c \rightarrow \infty$. In the resulting equation, divide both sides by $\left(q^{k} ; q^{k}\right)_{s}$.

We now conclude the proof of (5), using Corollary 1.1 in the last line. With 


$$
\begin{aligned}
N & =n_{1}+n_{2}+\cdots+n_{k-1}, \\
\left(-a q^{k} ; q^{k}\right)_{\infty} \sum_{n_{1}, \ldots, n_{k-1}>0} \frac{a^{N} q^{k N(N-1) / 2+n_{1}+2 n_{2}+\cdots+(k-1) n_{k-1}}}{\left(q^{k} ; q^{k}\right)_{n_{1}} \cdots\left(q^{k} ; q^{k}\right)_{n_{k-1}}\left(-a q^{k} ; q^{k}\right)_{N}} & \sum_{n_{1}, \ldots, n_{k-1}>0} \frac{a^{N} q^{k N(N-1) / 2+n_{1}+2 n_{1}+2 n_{2}+\cdots+(k-1) n_{k-1}}}{\left(q^{k} ; q^{k}\right)_{n_{1}} \cdots\left(q^{k} ; q^{k}\right)_{n_{k-1}}}\left(-a q^{k+k N} ; q^{k}\right)_{\infty} \\
& =\sum_{n_{1}, \ldots, n_{k-1}>0} \sum_{n_{k}>0} \frac{a^{N+n_{k}} q^{k\left(N+n_{k}\right)\left(N+n_{k}-1\right) / 2+n_{1}+\cdots+(k-1) n_{k-1}+k n_{k}}}{\left(q^{k} ; q^{k}\right)_{n_{1}} \cdots\left(q^{k} ; q^{k}\right)_{n_{k-1}}\left(q^{k} ; q^{k}\right)_{n_{k}}} \\
& =\sum_{s>0} a^{s} q^{s+k s(s-1) / 2} \sum_{m_{1}=0}^{s} \sum_{m_{2}=0}^{s-m_{1}} \cdots \sum_{m_{k-1}=0}^{s-\left(m_{1}+\cdots+m_{k-2}\right)} \\
& =\sum_{s>0} \frac{a^{s} q^{s+k s(s-1) / 2}}{(q)_{s}} \cdot q^{m_{1}+2 m_{2}+\cdots+(k-1) m_{k-1}}
\end{aligned}
$$

Andrews and Askey [5] have demonstrated that if we set $a=1$ in equation (3), the resulting equation has the partition theoretic interpretation: The number of partitions of $n$ with minimal difference 2 is equal to the number of partitions of $n$ into distinct parts such that the smallest even part is greater than twice the number of odd parts. Using their methods on equation (5) with $a=1$ gives us:

COROLlaRY 1.2. The number of partitions of $n$ with minimal difference $k$ is equal to the number of partitions of $n$ into distinct parts such that for $1 \leqslant i \leqslant k$, the smallest part congruent to $i(\bmod k)$ is larger than $k \sum_{j=1}^{i-1} r(j)$, where $r(j)$ is the number of parts congruent to $j(\bmod k)$.

This is a result of the author's [7]. It was previously proved by establishing the correspondence between partitions.

ProOF OF (6).

Proposition 2.

$$
\begin{gathered}
\Phi_{D}[a ; \overbrace{\left.b, \ldots, b ; a q / b ; q ; \zeta q / b, \zeta^{2} q / b, \ldots, \zeta^{k-1} q / b\right]}^{k-1} \\
=\frac{(a)_{\infty}(q / b)_{\infty}\left(q^{k} ; q^{k}\right)_{\infty}\left(a q^{k} / b^{k} ; q^{k}\right)_{\infty}}{(q)_{\infty}(a q / b)_{\infty}\left(a ; q^{k}\right)_{\infty}\left(q^{k} / b^{k} ; q^{k}\right)_{\infty}},
\end{gathered}
$$

where $\zeta=e^{2 \pi i / k}$.

Proof. In (1), let $n=k-1, \alpha=a, \beta_{1}=\cdots=\beta_{k-1}=b, \gamma=a q / b$, $x_{1}=\zeta q / b, x_{2}=\zeta^{2} q / b, \ldots, x_{k-1}=\zeta^{k-1} q / b$; then 
ANDREWS' BASIC LAURICELLA TRANSFORMATION

93

$$
\begin{aligned}
\Phi_{D}\left[a ; b, \ldots, b ; a q / b ; q ; \zeta q / b, \zeta^{2} q / b, \ldots, \zeta^{k-1} q / b\right] \\
\quad=\frac{(a)_{\infty}}{(a q / b)_{\infty}} \prod_{i=1}^{k-1} \frac{\left(\zeta^{i} q\right)_{\infty}}{\left(\zeta^{i} q / b\right)_{\infty}}{ }_{k} \phi_{k-1}\left[\begin{array}{c}
\left.q / b, \zeta q / b, \ldots, \zeta^{k-1} q / b ; q, a\right] \\
\zeta q, \ldots, \zeta^{k-1} q
\end{array}\right] \\
\quad=\frac{(a)_{\infty}}{(a q / b)_{\infty}} \frac{\left(q^{k} ; q^{k}\right)_{\infty}(q / b)_{\infty}}{(q)_{\infty}\left(q^{k} / b^{k} ; q^{k}\right)_{\infty}} \sum_{n>0} \frac{\left(q^{k} / b^{k} ; q^{k}\right)_{n}}{\left(q^{k} ; q^{k}\right)_{n}} a^{n} \\
=\frac{(a)_{\infty}(q / b)_{\infty}\left(q^{k} ; q^{k}\right)_{\infty}\left(a q^{k} / b^{k} ; q^{k}\right)_{\infty}}{(q)_{\infty}(a q / b)_{\infty}\left(a ; q^{k}\right)_{\infty}\left(q^{k} / b^{k} ; q^{k}\right)_{\infty}}
\end{aligned}
$$

(by Bailey [6, p. 66, (4)]).

This establishes Proposition 2. Note that for $k=2$, this becomes the $q$-analog of Kummer's Theorem: (see Baum [8], Andrews [1]).

$$
{ }_{2} \phi_{1}\left[\begin{array}{c}
a, b ; q,-q / b \\
q a / b
\end{array}\right]=\frac{\left(a q ; q^{2}\right)_{\infty}(-q)_{\infty}\left(q^{2} a / b^{2} ; q^{2}\right)_{\infty}}{(q a / b)_{\infty}(-q / b)_{\infty}}
$$

Corollary 2.1 .

$$
\begin{aligned}
\sum_{m_{1}=0}^{s} \sum_{m_{2}=0}^{s-m_{1}} \cdots \sum_{m_{k-1}=0}^{s-\left(m_{1}+\cdots+m_{k-2}\right)} \frac{\zeta^{m_{1}+2 m_{2}+\cdots+(k-1) m_{k-1}}}{(q)_{m_{1}} \cdots(q)_{m_{k-1}}(q)_{s-\left(m_{1}+\cdots+m_{k-1}\right)}} \\
=\left\{\begin{array}{lll}
0, & \text { if } k \nmid s, & \text { where } \zeta=e^{2 \pi i / k} . \\
\frac{1}{\left(q^{k} ; q^{k}\right)_{s / k}}, & \text { if } k \mid s,
\end{array}\right.
\end{aligned}
$$

Proof. In Proposition 2, set $a=q^{-s}$ and let $b \rightarrow 0$. In the resulting equation, divide both sides by $(q)_{s}$.

For $k=2$, Corollary 2.1 is a theorem of Gauss.

We now conclude the proof of (6), using Corollary 2.1 with $q$ replaced by $q^{2}$ in the last line. As before, $\zeta=e^{2 \pi i / k}$ and $N=n_{1}+\cdots+n_{k-1}$. We have

$$
\begin{aligned}
& \left(-a q ; q^{2}\right)_{\infty} \sum_{n_{1}, \ldots, n_{k-1}>0} \frac{q^{N^{2}} a^{N \zeta^{n_{1}}+2 n_{2}+\cdots+(k-1) n_{k-1}}}{\left(q^{2} ; q^{2}\right)_{n_{1}} \cdots\left(q^{2} ; q^{2}\right)_{n_{k-1}}\left(-a q ; q^{2}\right)_{N}} \\
& =\sum_{n_{1}, \ldots, n_{k-1}>0} \frac{q^{N^{2}} a^{N \zeta^{n_{1}+2 n_{2}}+\cdots+(k-1) n_{k-1}}}{\left(q^{2} ; q^{2}\right)_{n_{1}} \cdots\left(q^{2} ; q^{2}\right)_{n_{k-1}}}\left(-a q^{2 N+1} ; q^{2}\right)_{\infty} \\
& =\sum_{n_{1}, \ldots, n_{k-1}>0} \sum_{n_{k}>0} \frac{q^{\left(N+n_{k}\right)^{2}} a^{N+n_{k} \zeta^{n_{1}+2 n_{2}}+\cdots+(k-1) n_{k-1}}}{\left(q^{2} ; q^{2}\right)_{n_{1}} \cdots\left(q^{2} ; q^{2}\right)_{n_{k-1}}\left(q^{2} ; q^{2}\right)_{n_{k}}}
\end{aligned}
$$




$$
\begin{aligned}
& =\sum_{s>0} q^{s^{2}} a^{s} \sum_{m_{1}=0}^{s} \sum_{m_{2}=0}^{s-m_{1}} \cdots \sum_{m_{k-1}=0}^{s-\left(m_{1}+\cdots+m_{k-2}\right)} \\
& \frac{\zeta^{m_{1}+2 m_{2}+\cdots+(k-1) m_{k-1}}}{\left.\left(q^{2} ; q^{2}\right)_{m_{1}} \cdots\left(q^{2} ; q^{2}\right)_{m_{k-1}}\left(q^{2} ; q^{2}\right)_{s-\left(m_{1}+\cdots+m_{k-1}\right)}\right)} \\
& =\sum_{s>0} \frac{q^{k^{2} s^{2}} a^{k s}}{\left(q^{2 k} ; q^{2 k}\right)_{s}} .
\end{aligned}
$$

This finishes the proof of (6).

ACKNOWLEDGEMENT. I wish to thank Richard Askey for his suggestion that I look for a functional proof of my partition identity.

\section{REFERENCES}

1. G. E. Andrews, On the q-analog of Kummer's theorem and applications, Duke Math. J. 40 (1973), 525-528.

2. Problems and prospects for basic hypergeometric functions, Theory and Application of Special Functions, edited by R. Askey, Academic Press, New York, 1975, pp. 191-224.

3. , q-identities of Auluck, Carlitz and Rogers, Duke Math. J. 33 (1966), 575-582.

4. Summations and transformations for basic Appell series, J. London Math. Soc. (2) 4 (1972), 618-622.

5. G. E. Andrews and R. Askey, Enumeration of partitions: the role of Eulerian series and q-orthogonal polynomials, Proc. of the NATO Advanced Study Institute "Higher Combinatorics", Berlin, Sept. 1-10, 1976, edited by M. Aigner, Reidel, Dordrecht, 1977.

6. W. N. Bailey, Generalized hypergeometric series, Cambridge Univ. Press, Cambridge, 1935.

7. D. M. Bressoud, A new family of partition identities, Pacific J. Math. (to appear).

8. J. A. Daum, The basic analog of Kummer's theorem, Bull. Amer. Math. Soc. 48 (1942), 711-713.

9. E. Heine, Handbuch der Kugelfunctionen, Vol. I, Reimer, Berlin, 1878.

10. L. J. Rogers, Second memoir on the expansion of certain infinite products, Proc. London Math. Soc. 25 (1894), 318-343.

11. G. N. Watson, A note on Lerch's functions, Quart. J. Math. 42 (1937), 272-304.

Department of Mathematics, Pennsylvania State University, University Park, PennSYLVANIA 16802 